\title{
Fuzzy-rough set models and fuzzy-rough data reduction
}

\author{
Alireza Mansouri Ghroutkhar ${ }^{1, *}$ and Hassan Mishmast $\mathrm{Nehi}^{1}$ \\ 1 Department of Mathematics, University of Sistan and Baluchestan, Zahedan, Iran \\ E-mail:〈Alirezamansourighroutkhar@gmail.com〉,〈hmnehi@hamoon.usb.ac.ir〉
}

\begin{abstract}
Rough set theory is a powerful tool to analysis the information systems. Fuzzy rough set is introduced as a fuzzy generalization of rough sets. This paper reviewed the most important contributions to the rough set theory, fuzzy rough set theory and their applications. In many real world situations, some of the attribute values for an object may be in the set-valued form. In this paper, to handle this problem, we present a more general approach to the fuzzification of rough sets. Specially, we define a broad family of fuzzy rough sets. This paper presents a new development for the rough set theory by incorporating the classical rough set theory and the interval-valued fuzzy sets. The proposed methods are illustrated by an numerical example on the real case.
\end{abstract}

Keywords: fuzzy rough set, lower approximation, upper approximation discernibility matrix

Received: August 04, 2019; accepted: June 18, 2020; available online: July 07, 2020

DOI: $10.17535 /$ crorr.2020.0006

\section{Introduction}

For the first time, rough set theory was proposed by Pawlak [21, 22, 23, 24]. Lateron, Dubois and Prade [6, 7] introduced fuzzy-rough set as a fuzzy generalization of rough sets. In this paper, we present a more general approach to the fuzzification of rough sets. specifically, we define a broad family of fuzzy-rough sets, each one of which, called an $(\mathcal{F}, \tau)$-fuzzy-rough set, is determined by an implicator $\mathcal{F}$ and a triangular norm $\tau$ [21]. Furthermore the contribution of current paper is to suggest a novel devlopment of the rough set theory using merging the classical Pawlak [21] rough-set theory with the interval-valued fuzzy set theory, i.e. the interval-valued (I-V) fuzzy informathion system which is defined by a binary interval-valued fuzzy relation $R \in F^{(i)}(U \times U)$ on the universe $U$. [9, 25, 28]

The presented method is applied to find the optimal subsets in the fuzzy-rough data reduction process. [3, 11, 28, 27]

Moreover, in many real world problems, some information in the data will be lost according to the tolerance relation $[2,3,8,13,27,28,35]$ or in many cases information is accessible in the form of data table known as information system (IS). Considering this, in this paper we suggest a fuzzy relation and construct a fuzzy rough set model for set-valued information system.

In recent years some researchers have tried to developed novel methods to deals with situations in which incomplete information are in form of fuzzy, rough or their combinations. Huang et al. [10] developed a new multigranulation rough set model through a combination of multigranulation rough sets with intuitionistic fuzzy rough sets called an intuitionistic fuzzy multigranulation rough set. Liang et al. [16] at first introduced incremental mechanisms for three representative information entropies when a group of objects are added to a decision table and then develop a group incremental rough feature selection algorithm based on information

${ }^{*}$ Corresponding author. 
entropy. An et al. [1] have proposed a data-distribution-aware FRS model that considers distribution information and incorporates it in computing lower and upper fuzzy approximations. The proposed model considers not only the similarity between samples, but also the probability density of classes. Sun et al. [29] defined the rough fuzzy set on a probabilistic approximation space over two universes and then defined the fuzzy probabilistic approximation over two universes by introducing a probability measure to the approximation space over two universes and, finally, established the fuzzy rough set model on the probabilistic approximation space over two universes. Zhao et al. [36] were the first researchers who proposed a nested structural classifier, called nested classifier. There are two main contributions in their work as: The first and the most important result is that a series nested structure on the basic concepts of rough classifiers, such as lower approximation, discenribility vector and covering vector, is discovered.

Another key result is that several algorithms to find nested discernibility vector, nested rule and nested covering vector are designed. Wang et al. [32] defined the fuzzy decision of a sample using the concept of fuzzy neighborhood. Then, a parameterized fuzzy relation is introduced to characterize the fuzzy information granules, using which the fuzzy lower and upper approximations of a decision are reconstructed and a new fuzzy rough set model is introduced. Yang et al. [34] have studied incremental attribute reduction with fuzzy rough sets and applying the incremental process, two incremental algorithms for attribute reduction with fuzzy rough sets are presented for one incoming sample and multiple incoming samples, respectively. Sun et al. [30] considered rough approximation of a fuzzy concept under the framework of multigranulation over two different universes of discourse, i.e. multigranulation fuzzy rough set models over two universes and presented three types of multigranulation fuzzy rough set over two universes by the constructive approach, respectively. Lin et al. [17] introduced fuzzy mutual information to evaluate the quality of features in multi-label learning, and have designed efficient algorithms to conduct multi-label feature selection when the feature space is completely known or partially known in advance. Lin et al. [19] proposed a novel fuzzy rough set model for attribute reduction in multi-label learning. The authors at first defined the score vector of each sample to evaluate the probability of being different class's sample with respect to the target sample, and then, local sampling is leveraged to construct a robust distance between samples. Wang et al. [31] introduced distance measures into fuzzy rough sets and proposed a novel method for attribute reduction. Wang et al. [31] at first constructed a fuzzy rough set model based on distance measure with a fixed parameter. Then, the fixed distance parameter is replaced by a variable one to better characterize attribute reduction with fuzzy rough sets. To select more effective feature genes, $\mathrm{Xu}$ et al. [33] proposed a new rough uncertainty metric model. To do this, Xu et al. [33] constructed the fuzzy neighborhood granule of the sample by combining the fuzzy similarity relation with the neighborhood radius in the rough set, and the rough decision has defined by using the fuzzy similarity relation and the decision equivalence class. Then, the fuzzy neighborhood granule and the rough decision have introduced into the conditional entropy, and the rough uncertainty metric model has proposed.

This paper is organized as follows. We first will review the basic of the fuzzy rough sets in Section 2. In Section 3, the interval-valued fuzzy information system is reviewed. Section 4 compares the interval-valued fuzzy rough set model with the other rough set models. In Section 5, data reduction of the interval-valued fuzzy information system is presented. Section 6 presents a Fuzzy rough set model for the data reduction of set-valued data. In Section 7 a numerical example is presented to illustrate the contribution of the current paper. Finally, the conclusion remarks of the paper are given in Section 8. 


\section{Fundementals of the fuzzy rough sets}

In this section we introduce the definitions of fuzzy rough approximations and fuzzy rough sets. Assume $X$ be a nonempty universe and assume that $\tilde{\mathrm{R}}$ be an equivalence relation on $\mathrm{X}$. The following notation will be used. Given a nonempty universe $X$, by $P(X)$ we will denote a power-set on $X$. The following notation will be used. Given a nonempty universe $X$, by $P(X)$ we will denote a power-set on $X$. If $R$ is an equivalence relation on $X$ then for every $x \in X,[x]_{R}$ stands for the equivalence class of $R$ with the representant $x$, i.e. $[x]_{R}=\{y \in X:(x, y) \in R\}$. Finally, for any $Z \subseteq X$, we write $Z$ to denote the complementation of $Z$ in $X$, that is the set $X \backslash Z$.

Definition 1. A pair $Z S=(X, R)$, where $X \neq \emptyset$ and $R$ is an equivalence relation on $X$ is called an approximation space.

Definition 2. For an approximation space $Z S=(X, R)$, by a rough approximation in $Z S$ we mean a mapping

$$
\operatorname{APr}_{Z s}: \mathcal{P}(X) \rightarrow \mathcal{P}(X) \times \mathcal{P}(X)
$$

defined by for every $Z \in \mathcal{P}(X), A P r_{Z s}(Z)=(\underline{Z S}(Z), \overline{Z S}(Z))$ where

$$
\begin{aligned}
& \underline{Z S}(Z)=\left\{x \in X ;[x]_{R} \subseteq Z\right\}, \\
& \overline{Z S}(Z)=\left\{x \in X ;[x]_{R} \cap Z \neq \emptyset\right\} .
\end{aligned}
$$

$\underline{Z S}(Z)$ is called a lower rough approximation of $Z$ in $Z S$. where as $\overline{Z S}(Z)$ is called an upper rough approximation of $Z$ in $Z s$.

Definition 3. Given an approximation space $Z S=(X, R)$, a pair $(L, U) \in \mathcal{P}(X) \times \mathcal{P}(X)$ is called a rough set in $Z s$ iff $(L, U)=A \operatorname{Pr}_{Z s}(Z)$ for some $Z \in \mathcal{P}(X)$.

For any approximation space $Z s=(X, R)$, a subset $Z \subseteq X$ is called definable in $Z s$ iff $\underline{Z s}(Z)=\overline{Z s}(Z)$.

\section{A review on the $\mathrm{I}-\mathrm{V}$ fuzzy information system}

Assume that $I=[0,1]$. Also, assume that $[I]=\{[\alpha, \beta]: \alpha \leq \beta, \alpha, \beta \in I\}$. for any $\alpha \in I$, define $\bar{\alpha}=[\alpha, \alpha]$. obviously, $\alpha \in[I]$.

Definition 4. If $\forall \alpha_{i} \in I, i \in J$, we define

$$
\begin{aligned}
& \max _{i \in J} \alpha_{i}=\sup \left\{\alpha_{i}: i \in J\right\}, \\
& \min _{i \in J} \alpha_{i}=\inf \left\{\alpha_{i}: i \in J\right\}, \\
& \max _{i \in J}\left[\alpha_{i}, \beta_{i}\right]=\left[\max _{i \in J} \alpha_{i}, \max _{i \in J} \beta_{i}\right], \\
& \min _{i \in J}\left[\alpha_{i}, \beta_{i}\right]=\left[\min _{i \in J} \alpha_{i}, \min _{i \in J} \beta_{i}\right] .
\end{aligned}
$$

Particular for $\left[\alpha_{i}, \beta_{i}\right] \in[I], i=1,2$, we define

$$
\begin{aligned}
& {\left[\alpha_{i} \beta_{i}\right]=\left[\alpha_{2}, \beta_{2}\right] \text { iff } \alpha_{1}=\alpha_{2}, \beta_{1}=\beta_{2}} \\
& {\left[\alpha_{1}, \beta_{1}\right] \leq\left[\alpha_{2}, \beta_{2}\right] \text { iff } \alpha_{1} \leq \alpha_{2}, \beta_{1} \leq \beta_{2}} \\
& {\left[\alpha_{1}, \beta_{1}\right]<\left[\alpha_{2}, \beta_{2}\right] \text { iff }\left[\alpha_{1}, \beta_{1}\right] \leq\left[\alpha_{2}, \beta_{2}\right] b u t\left[\alpha_{1}, \beta_{1}\right] \neq\left[\alpha_{2}, \beta_{2}\right]}
\end{aligned}
$$

Definition 5. Assume that $X$ be an ordinary non-empty set. Then the mapping $Z: X \rightarrow[I]$ is called an I-V fuzzy set in $X$. All I-V fuzzy set on $X$ are denoted as $F^{(i)}(X)$. 
Definition 6. If $Z \in F^{(i)}(X)$ Assume that $Z(x)=\left[Z^{-}(x), Z^{+}(x)\right]$. where $x \in X$, then two fuzzy sets $Z^{-}: X \rightarrow I$, and $Z^{+}: X \rightarrow I$ are called the lower fuzzy set and the upper fuzzy set about $Z$, respectively.

Definition 7. Assume that $Z \in F^{(i)}(X),\left[\lambda_{1}, \lambda_{2}\right] \in[I]$, we call $Z_{\left[\lambda_{1}, \lambda_{2}\right]}=\left\{x \in X ; Z^{-}(x) \geq\right.$ $\left.\lambda_{1}, Z^{+}(x) \geq \lambda_{2}\right\}$, and $Z_{\left(\lambda_{1}, \lambda_{2}\right)}=\left\{x \in X ; Z^{-}(x)>\lambda_{1}, Z^{+}(x)>\lambda_{2}\right\}$, the $\left[\lambda_{1}, \lambda_{2}\right]$-level set of $Z$ and $\left(\lambda_{1}, \lambda_{2}\right)$-level set of $Z$, respectively. Where $\left(\lambda_{1}, \lambda_{2}\right)$ in $Z_{\left(\lambda_{1}, \lambda_{2}\right)}$ is not an interval, it is only a sign, and we may admit $\lambda_{1}=\lambda_{2}$, clearly, $x \in Z_{\left[\lambda_{1}, \lambda_{2}\right]}$ iff $Z_{\left[\lambda_{1}, \lambda_{2}\right]} \geq\left[\lambda_{1}, \lambda_{2}\right]$.

Definition 8. Assume that $Z \in F^{(i)}(X),\left[\lambda_{1}, \lambda_{2}\right] \in[I]$, we define

$$
\left(\left[\lambda_{1}, \lambda_{2}\right] Z\right)(x)=\left[\lambda_{1}, \lambda_{2}\right] \min \left[Z^{-}(x), Z^{+}(x)\right] .
$$

\subsection{The rough approximation of a crisp set on the $I-V$ fuzzy informa- tion system}

Assume that $U$ be a non-empty finite universe. A binary I-V fuzzy subset $\tilde{R}$ of $U \times U$ is called an intertval-valued fuzzy relation in $U$.

Definition 9. Assume that $U$ be a non-empty finite universe. for the $I-V$ fuzzy relation $\tilde{R}$ $\left(\tilde{R} \in F^{(i)}(U \times U)\right)$ of the universe $U$ :

1. $\tilde{R}$ is reflexive, if $\tilde{R}(x, y)=\overline{1}, \forall x, y \in U$,

2. $\tilde{R}$ is symetric, if $\tilde{R}(x, y)=\tilde{R}(y, x), \forall x, y \in U$,

3. $\tilde{R}$ is transitive, if $\tilde{R}(x, z) \geq \tilde{R}(x, y) \min \tilde{R}(y, z) \forall x, y, z \in U$.

If the fuzzy relation $\tilde{R}$ is reflexive, symmetric and transitive, then $\tilde{R}$ is an I-V fuzzy equivalence relation.

Definition 10. Assume that $(U, \tilde{R})$ be an $I$ - $V$ fuzzy information system for any $x \in U$, call $[x]^{(i)}: U \rightarrow[x], y \rightarrow \tilde{R}(x, y)$ the $I-V$ fuzzy neighborhood of $x$. Assume that $U$ be a non-empty finite universe for any crisp set $X(X \subseteq U)$ of $U$, define

$$
\begin{aligned}
& \underline{\tilde{R}}(X)(y)=\min _{x \in X}(\overline{1}-\tilde{R}(x, y)), \\
& \overline{\tilde{R}}(X)(y)=\max _{x \in X} \tilde{R}(x, y) \text { for anyy } \in U .
\end{aligned}
$$

Obviously we can obtain following relationships:

$$
\begin{aligned}
& \underline{\tilde{R}}(X)(y)=\left[\min _{x \notin X}\left(1-\tilde{R}^{+}(x, y), \min _{x \notin X}\left(1-\tilde{R}^{-}(x, y)\right)\right],\right. \\
& \underline{\tilde{R}}(X)(y)=\left[\max _{x \in X} \tilde{R}^{-}(x, y), \max _{x \in X} \tilde{R}^{+}(x, y)\right] .
\end{aligned}
$$

Definition 11. Assume that $(U, \tilde{R})$ be an $I-V$ fuzzy information system and $Z$ be the $I-V$ fuzzy set of universe $U$. Define the $I-V$ fuzzy upper approximation $\underline{\tilde{R}}(Z)$ and the $I-V$ fuzzy upper approximation $\bar{R}(Z)$ of $Z$ in the $I-V$ fuzzy information system $(U, \tilde{R})$ as follows, respectively for any $x \in U$.

$$
\begin{aligned}
& \underline{\tilde{R}}(Z)(x)=\min \{Z(y) \max (\overline{1}-\tilde{R}(x, y)): y \in U\}, \\
& \overline{\tilde{R}}(Z)(x)=\max \{Z(y) \min \tilde{\tilde{R}}(x, y): y \in U\} .
\end{aligned}
$$


Clearly, the above definition implies equivalence of the following form:

$$
\begin{aligned}
\underline{\tilde{R}}(Z)(x) & =\min _{y \in U}(Z(y) \max (i-\tilde{R}(x, y)) \\
& =\left[\min _{y \in U}\left(Z^{-}(y) \max \left(1-\tilde{R}^{+}(x, y)\right), \min \left(Z^{+}(y) \max \left(1-\tilde{R}^{-}(x, y)\right)\right)\right]\right. \\
& \forall x \in U, \\
\tilde{\tilde{R}}(Z)(x) & =\max _{y \in U}(Z(y) \min \tilde{R}(x, y)) \\
& =\left[\max _{y \in U}\left(Z^{-}(y) \min \tilde{R}^{-}(x, y)\right), \max _{y \in U}\left(Z^{+}(y) \min ^{+}(x, y)\right)\right], \forall x \in U .
\end{aligned}
$$

\section{Comparision of the $\mathrm{I}-\mathrm{V}$ fuzzy rough set model with other models}

In this section, we will review the relationships between the I-V fuzzy rough set with the other classical pawlak [21] rought set model by changing the relations and the subsets of the universe $U$. It is trivial to show that the I-V fuzzy rough set model is an development of the classical pawlak [21] $\tilde{R} S$ model.

Case 1. If $Z \in F(U), \tilde{R} \in F^{(i)}(U \times U)$. For any $a \in[0,1]$ write $\bar{a}=[a, a] \in[I]$ then

$$
\begin{aligned}
\underline{\tilde{R}}(Z)(x) & =\min _{y \in U}\left(Z(y) \max \left(1^{-}-\tilde{R}(x, y)\right)\right) \\
& \min _{y \in U}[Z(y), Z(y)] \max (\overline{1}-\tilde{R}(x, y)) \\
& =\left[\min _{y \in U}\left(Z(y) \max \left(1-\tilde{R}^{+}(x, y)\right)\right), \min _{y \in U}\left(Z(y), \max \left(1-\tilde{R}^{-}(x, y)\right)\right]\right. \\
\tilde{\tilde{R}}(Z)(x) & =\max _{y \in U} Z(y) \min \tilde{R}(x, y) \\
& =\max _{y \in U}\left(Z(y) \min \left[\tilde{R}^{-}(x, y), \tilde{R}^{+}(x, y)\right]\right) \\
& =\max _{y \in U}\left([Z(y), Z(y)] \min \left[\tilde{R}^{-}(x, y), \tilde{R}^{+}(x, y)\right]\right) \\
& =\left[\max _{y \in U}\left(Z(y) \min \tilde{R}^{-}(x, y)\right), \max _{y \in U}\left(Z(y) \min ^{+}(x, y)\right)\right]
\end{aligned}
$$

for any $x \in U$.

Case 2. If $Z \in F^{(i)}(U), \tilde{R} \in F(U \times U)$, for any $x \in U$ :

$$
\begin{aligned}
& \underline{\tilde{R}}(Z)(x)=\left[\min _{y \in U}\left(Z^{-}(y) \max (1-\tilde{R}(x, y))\right), \min _{y \in U}\left(Z^{+}(y) \max (1-\tilde{R}(x, y))\right)\right] \\
& \tilde{\tilde{R}}(Z)(x)=\left[\max _{y \in U}\left(Z^{-}(y) \min (1-\tilde{R}(x, y))\right), \max _{y \in U}\left(Z^{+}(y) \min (1-\tilde{R}(x, y))\right)\right]
\end{aligned}
$$

Case 3. If $Z \in F^{(i)}(U), \tilde{R} \subseteq U \times U$, for any $y \in U$, we have $y \in[x]_{\tilde{R}}$, then $\tilde{R}(x, y)=1$. Therefor, for any $x \in U$ :

$$
\begin{aligned}
& \underline{\tilde{R}}(Z)(x)=\left[\operatorname { m i n } _ { y \in U } \left(Z^{-}(y) \max (1-1), \min _{y \in U}\left(Z^{+}(y) \max (1-1)\right]\right.\right. \\
& =\min _{y \in U}\left[Z^{-}(y), Z^{+}(y)\right] \\
& =\left[\min _{y \in U} Z^{-}(y), \min _{y \in U} Z^{+}(y)\right] \\
\tilde{\tilde{R}}(Z)(x) & =\left[\max _{y \in U}\left(Z^{-}(y) \min 1\right), \max _{y \in U}\left(Z^{+}(y) \min 1\right)\right] \\
& =\left[\max _{y \in U} Z^{-}(y), \max _{y \in U} Z^{+}(y)\right]
\end{aligned}
$$


Case 4. If $Z \subseteq U, \tilde{R} \in F^{(i)}(U \times U)$ Then for any $x \in U$

$$
\begin{aligned}
\underline{\tilde{R}}(Z)(x) & =\min \{Z(y) \max (\overline{1}-\tilde{R}(x, y)): y \in U\} \\
& =\min _{y \notin Z}(\overline{1}-\tilde{R}(x, y)) \\
& {\left[\min _{y \notin Z}\left(\overline{1}-\tilde{R}^{+}(x, y)\right), \min _{y \notin Z}\left(\overline{1}-\tilde{R}^{-}(x, y)\right)\right] } \\
\overline{\tilde{R}}(Z)(x) & =\max _{2}\{Z(y) \min \tilde{R}(x, y): y \in U\} \\
& =\max _{y \in Z} \tilde{R}(x, y) \\
& =\left[\max _{y \in Z} \tilde{R}^{-}(x, y), \max _{y \in Z} \tilde{R}^{+}(x, y)\right]
\end{aligned}
$$

Case 5. If $Z \in F(U), \tilde{R} \in F(U \times U)$ Then for any $x \in U$ we have:

$$
\begin{aligned}
\underline{\tilde{R}}(Z)(x) & =\min \{Z(y) \max (\overline{1}-\tilde{R}(x, y)): y \in U\} \\
& =\min \{Z(y) \max (1-\tilde{R}(x, y): y \in U\} \\
\overline{\tilde{R}}(Z)(x) & =\max \{Z(y) \min \tilde{R}(x, y): y \in U\}
\end{aligned}
$$

Case 6. If $Z \subseteq U, \tilde{R} \in F(U \times U)$ Then for any $x \in U$ we have:

$$
\begin{aligned}
& \underline{\tilde{R}}(Z)(x)=\min \{Z(y) \max (\overline{1}-\tilde{R}(x, y)): y \in U\} \\
&=\min \{Z(y) \max (1-\tilde{R}(x, y): y \in U\} \\
&=\min _{y \notin Z}(1-\tilde{R}(x, y)) \\
& \overline{\tilde{R}}(Z)(x)=\max \{Z(y) \min \tilde{R}(x, y): y \in U\} \\
& \max _{y \in Z} \tilde{R}(x, y)
\end{aligned}
$$

Case 7. If $Z \subseteq U, \tilde{R} \subseteq U \times U$ then for any $x \in U$ we have:

$$
\underline{\tilde{R}}(Z)(x)=1 \Leftrightarrow \forall y \in U,
$$

then there is

$$
\begin{aligned}
& Z(y) \max (\overline{1}-\tilde{R}(x, y)=Z(y) \max (1-\tilde{R}(x, y))=1 \\
& \Leftrightarrow \forall y \in U, y \notin Z \text { implicates the }(x, y) \notin \tilde{\tilde{R}} \\
& \Leftrightarrow \forall y \notin Z \text { implicates the } y \notin[x]_{\tilde{R}} \\
& \Leftrightarrow[x]_{\tilde{R} \subseteq Z} \\
& \overline{\tilde{R}}(Z)(x)=1 \Leftrightarrow \exists y \in U, \text { so } Z(y)=1 \text { and } \tilde{R}(x, y)=1 \text { is holding } \\
& \Leftrightarrow Z \cap[x]_{\tilde{R}} \neq \emptyset
\end{aligned}
$$


Case 8. If $Z \in F^{(i)}(U), \tilde{R} \subseteq P(U \times U)$. That is, $\tilde{R}(x, y)=\tilde{R}_{s}(x)=\{y \in U:(x, y) \in \tilde{R}\}$. Then for any $y \in U$. if $y \in \tilde{R}_{s}(x)$, then $\tilde{R}(x, y)=1$. Therefor, for any $x \in U$

$$
\begin{aligned}
& \underline{\tilde{R}}(Z)(x)=\min \left\{Z(y) \max (\overline{1}-\tilde{R}(x, y)): y \in \tilde{R}_{s}(x)\right\} \\
& =\min \left\{Z(y) \max (\overline{1}-1): y \in \tilde{R}_{s}(x)\right\} \\
& =\left[\min _{y \in U}\left(Z^{-}(y) \max (1-1), \min _{y \in U}\left(Z^{+}(y) \max (1-1)\right)\right\}\right. \\
& =\min _{y \in U}\left[Z^{-}(y), Z^{+}(y)\right] \\
& =\left[\min _{y \in U} Z^{-}(y), Z_{y \in U} Z^{+}(y)\right] \\
& =\left[\min \left\{Z^{-}(y): y \in \tilde{R}_{s}(x)\right\}, \min \left\{Z^{+}(y): y \in \tilde{R}_{s}(x)\right\}\right] \\
& \underline{\operatorname{apr}}(Z)(x) \\
& \overline{\tilde{R}}(Z)(x)=\max \{Z(y) \min \tilde{R}(x, y): y \in U\} \\
& =\max \left\{Z(y) \min \tilde{R}(x, y): y \in \tilde{R}_{s}(x)\right\} \\
& =\max \left\{Z(y) \min \overline{1}: y \in \tilde{R}_{s}(x)\right\} \\
& =\left[\max _{y \in U}\left(Z^{-}(y) \min 1\right), \max _{y \in U}\left(Z^{+}(y) \min 1\right)\right] \\
& =\max _{y \in U}\left[Z^{-}(y), Z^{+}(y)\right] \\
& =\left[\max _{y \in U} Z^{-}(y), \max _{y \in U} Z^{+}(y)\right] \\
& =\left[\max \left\{Z^{-}(y): y \in \tilde{R}_{S}(x)\right\}, \max \left\{Z^{+}(y): y \in \tilde{R}_{s}(x)\right\}\right] \\
& =\overline{\operatorname{apr}}(Z)(x) \text {. }
\end{aligned}
$$

\section{Data reduction of the $\mathrm{I}-\mathrm{V}$ fuzzy information system}

Assume that $(U, Z, F)$ be an inbformation or database system. Here $U$ is the set of objects, i.e. $U=\left\{x_{1}, x_{2}, \ldots, x_{n}\right\}$.

Every element $x_{i}(i \leq n)$ in $U$ is called an object, and $Z$ is the attribute set, i.e. $Z=$ $\left\{a_{1}, a_{2}, \ldots, a_{m}\right\}$. every element $a_{j}(j \leq m)$ in $Z$ is an attribute, $F$ is the relation set of $U$ and $Z$, i.e. $F=\left\{f_{j}: j \leq m\right\},\left(f_{j}: U \rightarrow v_{j},(j \leq m)\right)$ and $v_{j}$ is the domain of the attribute $a_{j}$.

Definition 12. Assume that $(U, Z, F)$ be a classical information system, for any subset $B(B \subseteq$ $Z)$. $B$ is called the $I-V$ fuzzy reduction of the classical information system $(U, Z, F)$, if $B$ is the minimum set in the inclusion set which satisfies the following relations:

$$
\begin{aligned}
& \underline{\tilde{R}}_{Z}(X)(x)=\underline{\tilde{R}}_{B}(X)(x), \\
& \tilde{\tilde{R}}_{Z}(X)(x)=\overline{\tilde{R}}_{B}(X)(x),
\end{aligned}
$$

for any $X \in F^{(i)}(U)$ and $\forall x \in U$ where $\underline{\tilde{R}}_{Z}(X)(x), \underline{\tilde{R}}_{B}(X)(x), \overline{\tilde{R}}_{Z}(X)(x), \overline{\tilde{R}}_{B}(X)(x)$ are defined as the I-V rough fuzzy set.

$B$ is called the I-V fuzzy lower and upper approximation reduction of the classical information system $(U, Z, F)$ if $B$ be the minimum set that satisfies the following relations, respectively:

$$
\underline{\tilde{R}}_{Z}(X)(x)=\underline{\tilde{R}}_{B}(X)(x) \text { for any } X \in F^{(i)}(U), x \in U,
$$

and

$$
\overline{\tilde{R}}_{Z}(X)(x)=\overline{\tilde{R}}_{B}(X)(x) \text { for any } X \in F^{(i)}(U), x \in U .
$$

We call $(U, Z, F, D, G)$ an information system or decision table, where $(U, Z, F)$ information system the classical information system, $Z$ is the condition attribute set and $D$ the decision 
attribute set, i.e., $D=\left\{d_{1}, d_{2}, \ldots, d_{p}\right\}$. $G$ is the relation set of the $U$ and $D, G=\left\{g_{j}: j \leq p\right\}$ (where $g_{j}: U \rightarrow v_{j}^{\prime},(j \leq p)$ ), $v_{j}^{\prime}$ is the domain of the decision attribute $d_{j}$.

The information system $(U, Z, F, D, G)$ is called a consistent information system or called an inconsistent information system if $\tilde{R}_{Z} \subseteq \tilde{R}_{D}$ i.e., $U / \tilde{R}_{Z} \leq U / \tilde{R}_{D}$, (or $\forall x \in U$, for any $[x]_{Z}$, there exists $[x]_{D}$ such that the $[x]_{Z} \subseteq[x]_{D}$.)

$(U, Z, F, D, G)$ is called an I-V fuzzy information system, where $(U, Z, F)$ is the classical information system $\tilde{D}_{\tilde{D}}=\left\{\tilde{D}_{k}: k=1,2, \ldots, n\right\}, \tilde{D}_{k}$ is the I-V fuzzy sets of $U$, and $G$ the relation set of $U$ and $\tilde{D}$.

Definition 13. Assume that $(U, Z, F, \tilde{D})$ be the $I$ - $V$ fuzzy information system, for any $B \subseteq Z$, if we have:

$$
\underline{\tilde{R}}_{B}\left(\tilde{D}_{i}\right)(x)>\underline{\tilde{R}}_{B}\left(\tilde{D}_{j}\right)(x) \Leftrightarrow \underline{\tilde{R}}_{Z}\left(\tilde{D}_{i}\right)(x)>\underline{\tilde{R}}_{Z}\left(\tilde{D}_{j}\right)(x) \quad(i \neq j)
$$

$B$ is called the reduction of the $\mathrm{I}-\mathrm{V}$ fuzzy information system $(U, Z, F, \tilde{D})$ If $B$ be the minimum consistent set of $Z$ in the inclusion set.

In the next section, by introducing the discernibility matrix we present the knowledge reduction of the I-V fuzzy information system.

Assume that $(U, Z, F, \tilde{D})$ be the I-V fuzzy information system, $\tilde{R}_{Z}$ be the equivalence classes which induced by the condition attribute set $Z$, and universe is divided by $\tilde{R}_{Z}$ as following:

$U / \tilde{R}_{Z}=\left\{X_{1}, X_{2}, \ldots, X_{k}\right\}$, denoted as:

$$
\underline{\tilde{R}}_{Z}(\tilde{D})\left(X_{i}\right)=\left(\underline{\tilde{R}}_{Z}\left(\tilde{D}_{1}\right)\left(X_{i}\right), \underline{\tilde{R}}_{Z}\left(\tilde{D}_{2}\right)\left(X_{i}\right), \ldots, \underline{\tilde{R}}_{Z}\left(\tilde{D}_{r}\right)\left(X_{i}\right)\right) .
$$

Definition 14. Assume that $(U, Z, F, \tilde{D})$ be I-V fuzzy information system.

$$
D_{i j}= \begin{cases}\left\{a_{k} \in Z: f_{l}\left(X_{i}\right) \neq f_{l}\left(X_{j}\right)\right\} & g_{X_{i}}\left(\tilde{D}_{k}\right) \neq g_{X_{j}}\left(\tilde{D}_{k}\right) \\ Z & g_{X_{i}}\left(\tilde{D}_{k}\right)=g_{X_{j}}\left(\tilde{D}_{k}\right)\end{cases}
$$

is called the discernibility matrix of $(U, Z, F, \tilde{D})$ (where $g_{X_{i}}\left(\tilde{D}_{k}\right)$ denotes the maximum value of $\underline{\tilde{R}}_{Z}(\tilde{D})\left(X_{i}\right)$ at the line of $k$, i.e., the rows $i$ and $j$ of $\left.E q .\left(^{*}\right)\right)$.

Theorem 1. Assume that $(U, Z, F, \tilde{D})$ be the $I$ - $V$ fuzzy information system. If there exists a subset $B \subseteq Z$ such that $B \cap D_{i j} \neq \emptyset(i, j \leq k)$ then $B$ is the consistent set of $Z$.

Proof. See [2]

\section{A Fuzzy $\tilde{R} S$ model for the data reduction of (s-v) data}

In this section using the theory of Fuzzy sets and Rough sets we proposed a method to data reduction and information.

Definition 15. For a (s-v) information system $(U, Z, V, f) \forall b \in Z$, a tolerance relation is defined as:

$$
T_{b}=\{(x, y) \mid b(x) \cap b(y) \neq \emptyset\}
$$

For $B \subseteq Z$, a tolerance relation is defined as:

$$
T_{B}=\{(x, y) \mid \forall b \in B, b(x) \cap b(y) \neq \emptyset\}=\cap_{b \in B} T_{b}
$$

when $(x, y) \in T_{B}, x$ and $y$ are called indiscernible with respect to $B$, or we say that $x$ is tolerant with $y$ with respect to $B$. 
Example 1. Assume that $(U, Z, V, f)$ be a (s-v) information system. Assume that $b \in Z$ be an attribute and Assume that $x, y, z \in U$ be three objects. Assume that $b(x)=\left\{e_{1}, e_{2}, \ldots, e_{8}\right\}$, $b(y)=\left\{e_{8}, e_{9}, e_{15}\right\}$ and $b(z)=\left\{e_{1}, e_{2}, \ldots, e_{7}\right\}$. Then by Definition, we know that both $(x, y)$ and $(x, z)$ belong to $T_{b}$.

Definition 16. For the (s-v) information system $(U, Z, V, f), \forall b \in Z$, a fuzzy relation $\tilde{R}_{b}$ can be defined as

$$
\mu_{\tilde{R}_{b}}(x, y)=\frac{|b(x) \cap b(y)|}{|b(x) \cup b(y)|}
$$

For a set of attribute $B \subseteq Z$, a fuzzy relation $\tilde{R}_{B}$ is defined as

$$
\mu_{\tilde{R}_{B}}(x, y)=\inf _{b \in B} \mu_{\tilde{R}_{b}}(x, y)
$$

Two important features of the fuzzy relation are as follows:

1. since $\mu_{\tilde{R}_{b}}(x, y)=|b(x) \cap b(y)| /|b(x) \cup b(y)|=1$.

2. From $\mu_{\tilde{R}_{b}}(x, y)=\mu_{\tilde{R}_{b}}(y, x)=|b(x) \cap b(y)| /|b(x) \cup b(y)|$.

As we know $\tilde{R}_{b}$ is reflective and symmetric.

Hence, $\tilde{R}_{b}$ is a fuzzy tolerance relation.

Example 2. Assume that $(U, Z, V, f)$ be a (s-v) information system Assume that $b \in Z$ be an attribute and Assume that $x, y, z \in U$ be three objects

Assume that $b(x)=\left\{e_{1}, e_{2}, \ldots, e_{8}\right\}, b(y)=\left\{e_{8}, e_{9}, \ldots, e_{15}\right\}, b(z)=\left\{e_{1}, e_{2}, \ldots, e_{7}\right\}$ thus, we have

$$
\begin{aligned}
& \mu_{\tilde{R}_{b}}(x, y)=\frac{|b(x) \cap b(y)|}{|b(x) \cup b(y)|}=\frac{1}{15} \\
& \mu_{\tilde{R}_{b}}(x, z)=\frac{|b(x) \cap b(z)|}{|b(x) \cup b(z)|}=\frac{7}{8} .
\end{aligned}
$$

This example shows that the fuzzy tolerance relation retains more information than the crisp tolerance relation.

\subsection{Reduct, core and discernibility matrix of (s-v) information system}

Definition 17. For a (s-v) information system $S=(U, C, V, f), P \subseteq C$ is a rduct of $c$ iff

1. $\forall x, y \in U, \tilde{R}_{c}(x, y)=\tilde{R}_{p}(x, y)$

2. For any $P^{\prime} \subset P, \exists x, y \in U, \tilde{R}_{c}(x, y) \neq \tilde{R}_{P^{\prime}}(x, y)$

where $\tilde{R}_{C}, \tilde{R}_{P}$, and $\tilde{R}_{P^{\prime}}$ are defined by the method in Definition 22.

By definition, a reduct is an attribute set that induces the same fuzzy relation $\tilde{R}$ as the whole attribute set $c$. The set of all reducts of $c$ is denoted by $\operatorname{Red}(C)$.

Definition 18. For a (s-v) information system $S=(U, C, V, f), P \subseteq C$ is a reduct of $C$ in the framework of rough set model iff

1. $\forall x \in U, T_{c}(x, y)=T_{p}(x, y)$ 
2. For any $P^{\prime} \subset P, \exists x, y \in U, T_{c}(x, y) \neq T_{P}(x, y)$

Definition 19. Assume that informationsystem $=(U, Z, V, f)$ be a (s-v) information system for a set of relations $\left\{\tilde{R}_{a} \mid a \in C\right\}$ for attribute set $C$ and universe set $U$, the discernibility matrix $M$ is defined as

$$
M_{n \times n}=\left(M_{i j}\right)_{n \times n}=\left[\begin{array}{cccc}
M_{11} & M_{12} & \ldots & M_{1 n} \\
M_{21} & M_{22} & \ldots & M_{2 n} \\
\vdots & & \ddots & \\
M_{n 1} & M_{n 2} & \ldots & M_{n n}
\end{array}\right]
$$

where $n=|U|$ and $M_{i j}$ is a set of attributes $\forall a \in Z, a \in M_{i j}$ iff $\tilde{R}_{a}\left(u_{i}, u_{j}\right)=\tilde{R}_{c}\left(u_{i}, u_{j}\right)\left(u_{i}, u_{j} \in\right.$ $U$ )

From the definition $19, M_{i j}$ consist of all the attributes that lead to a minimal degree of indiscernibility between elements $u_{i} \in U$ and $u_{j} \in U$. In other words, $b \in M_{i j}$ iff $\tilde{R}_{b}\left(u_{i}, u_{j}\right)=$ $\min _{a \in C} \tilde{R}_{a}\left(u_{i}, u_{j}\right)$ using the definition of discernibility matrix we can calculate a discernibility function.

Definition 20. Assume that informationsystem $=(U, Z, V, f)$ be a $(s-v)$ information system. $A$ discernibility function $f$ for informationsystem is a Boolean function of $m$ Boolean variables $c_{1}^{*}, c_{2}^{*}, \ldots, c_{m}^{*}$ corresponding to the attributes $c_{1}, c_{2}, \ldots, c_{m}$, respectively, and defined as

$$
f\left(c_{1}^{*}, c_{2}^{*}, \ldots, c_{m}^{*}\right)=\min \left\{\max M_{i j}: M_{i j} \in M_{n \times n}\right\}
$$

where $\max M_{i j}$ is the disjunction of all variables $c^{*}$ such that $a \in M_{i j}$ and min denotes conjunction.

\subsection{Relative reduct, relative core and discernibility matrix of (s-v) decision tables}

In the following, we consider (s-v) information system with decisions, that is, (s-v) DS or (s-v) decision tabled.

Definition 21. For a (s-v) information system $S=(U, C, V, f) P \subseteq C$ is a reduct of $C$ in the framework of rough set model iff.

1. $\forall x, y \in U$ if $d(x) \neq d(y)$ then $T_{C}(x, y)=T_{P}(x, y)$

2. For any $P^{\prime} \subset P, \exists x, y \in U, d(x) \neq d(y)$ and $T_{C}(x, y) \neq T_{P^{\prime}}(x, y)$

where $T_{C}, T_{P}$ and $T_{P^{\prime}}$ are defined by the method in Definition 1 The set of all reducts of $C$ is denoted by $\operatorname{Red}_{d}^{\prime}(C)$.

Definition 22. The intersection of all relative reducts is called the care of $C$ and denoted by core $_{d}(C)$ :

$$
\text { core }_{d}(C)=\cap \operatorname{Red}_{d}(C)
$$

Definition 23. Assume that $S=(U, C \cup\{d\}, V, f)$ be a (s-v) DS. For a set of relations $\left\{\tilde{R}_{a} \mid a \in C\right\}$ for attributes set $C$ and univerough sete set $U$, the discernibility matrix $M D$ is defined as

$$
M D_{n \times n}=\left(M D_{i j}\right)_{n \times n}=\left[\begin{array}{cccc}
M D_{11} & M D_{12} & \ldots & M D_{1 n} \\
M D_{21} & M D_{22} & \ldots & M D_{2 n} \\
\vdots & \vdots & \ddots & \vdots \\
M D_{n 1} & M D_{n 2} & \ldots & M D_{n n}
\end{array}\right]
$$


where $n=|U|$. For $u_{i}, u_{j} \in U$, if $d\left(u_{i}\right)=d\left(u_{j}\right)$, then $M_{i j}=\emptyset$ otherwise, if $d\left(u_{i}\right) \neq d\left(u_{j}\right)$, then $M_{i j}$ is a set of attributes and $c \in M D_{i j}$ iff $\tilde{R}_{c}\left(u_{i}, u_{j}\right)=\tilde{R}_{C}\left(u_{i}, u_{j}\right), M D_{i j}$ can be formally defined as

$$
M D_{i j}= \begin{cases}\left\{c \mid c \in C, \tilde{R}_{c}\left(u_{i}, u_{j}\right)=\tilde{R}_{C}\left(u_{i}, u_{j}\right)\right. & d\left(u_{i}\right) \neq d\left(u_{j}\right) \\ \emptyset & d\left(u_{i}\right)=d\left(u_{j}\right)\end{cases}
$$

\section{Application}

For the (s-v) DS shown in Table 1. We can obtain three fuzzy relations generated according to the three conditional attributes products, importes, and exports.

\begin{tabular}{|c|llll|}
\hline Company & Products $\left(j_{1}\right)$ & Imports $\left(j_{2}\right)$ & Exportes $\left(j_{3}\right)$ & Prospects $(D)$ \\
\hline \hline Company 1 $\left(c_{1}\right)$ & $\{$ mp4, phone & $\{$ IMer1, IMer2 $\}$ & $\{$ EM1 & Good \\
Company 2 $\left(c_{2}\right)$ & $\{$ video,mp4 & $\{$ IMer2, IMer3 $\}$ & $\{$ EM2, EM3 $\}$ & Bad \\
Company 3 $\left(c_{3}\right)$ & $\{$ mp4 & $\{$ IMer1, IMer2 & $\{$ EM2 $\}$ & Bad \\
Company 4 $\left(c_{4}\right)$ & $\{$ mp4, phone $\}$ & $\{$ IMer1 IMer 2 $\}$ & $\{$ EM1, EM2 $\}$ & Good \\
Company 5 $\left(c_{5}\right)$ & $\{$ video $\}$ & $\{$ IMer3 & $\{$ EM3 & Bad \\
\hline
\end{tabular}

Table 1: $A(s-v) D S$ comprising five compaines.

For example,

$$
\tilde{R}_{j_{1}}\left(c_{1}, c_{2}\right)=\mid\{m p 4, \text { phone }\} \cap\{\text { video, } m p 4\}|/|\{m p 4, \text { phone }\} \cup\{\text { video, } m p 4\} \mid=\frac{1}{3}=0.33 \text {. }
$$

Thus, the relation matrix as follows:

$$
\begin{aligned}
\tilde{R}_{\left\{e_{1}\right\}} & =\left(\begin{array}{cccccc}
\tilde{R}_{j_{1}} & c_{1} & c_{2} & c_{3} & c_{4} & c_{5} \\
c_{1} & 1 & 0.33 & 0.5 & 1 & 0 \\
c_{2} & 0.33 & 1 & 0.5 & 0.33 & 0.5 \\
c_{3} & 0.5 & 0.5 & 1 & 0.5 & 0 \\
c_{4} & 1 & 0.33 & 0.5 & 1 & 0 \\
c_{5} & 0 & 0.5 & 0 & 0 & 1
\end{array}\right), \\
R_{\left\{j_{2}\right\}} & =\left(\begin{array}{cccccc}
\tilde{R}_{j_{2}} & c_{1} & c_{2} & c_{3} & c_{4} & c_{5} \\
c_{1} & 1 & 0.33 & 1 & 1 & 0 \\
c_{2} & 0.33 & 1 & 0.33 & 0.33 & 0.5 \\
c_{3} & 1 & 0.3 & 1 & 1 & 0 \\
c_{4} & 1 & 0.3 & 1 & 1 & 0 \\
c_{5} & 0 & 0.5 & 0 & 0 & 1
\end{array}\right), \\
\tilde{R}_{\left\{j_{3}\right\}} & =\left(\begin{array}{ccccccc}
\tilde{R}_{j_{3}} & c_{1} & c_{2} & c_{3} & c_{4} & c_{5} \\
c_{1} & 1 & 0.5 & 0 & 0.5 & 0 \\
c_{2} & 0.5 & 1 & 0 & 0.33 & 0.5 \\
u_{3} & 0 & 0 & 1 & 0.5 & 0 \\
c_{4} & 0.5 & 0.33 & 0.5 & 1 & 0 \\
c_{5} & 0 & 0.65 & 0 & 0 & 1
\end{array}\right) .
\end{aligned}
$$


As one can see in Table 1 for the (s-v) information system we can calculate the discernibility matrix:

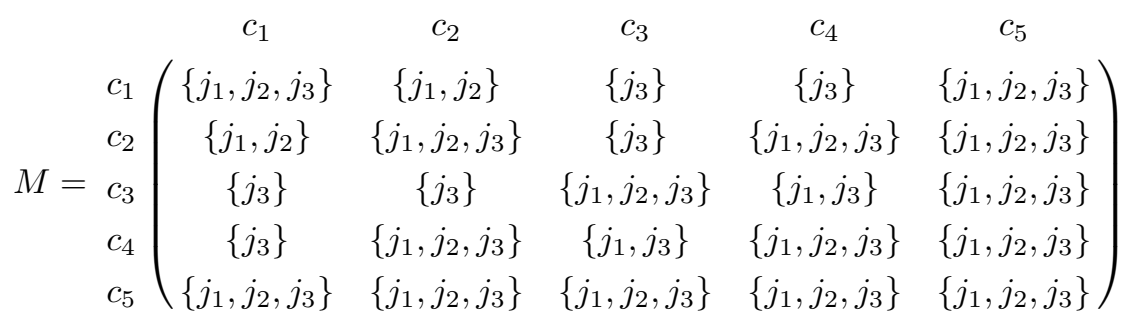

from wich we obtain the discernibility function:

$$
\begin{aligned}
f\left(j_{1}^{*}, j_{2}^{*}, j_{3}^{*}\right) & =\left(j_{1}^{*} \vee j_{2}^{*} \vee j_{3}^{*}\right) \wedge\left(j_{1}^{*} \vee j_{2}^{*}\right) \wedge\left(j_{3}^{*}\right) \wedge\left(j_{3}^{*}\right) \wedge\left(j_{1}^{*} \vee j_{2}^{*}, j_{3}^{*}\right) \\
& \wedge\left(j_{1}^{*} \vee j_{2}^{*}\right) \wedge\left(j_{1}^{*} \vee j_{2}^{*} \vee j_{3}^{*}\right) \wedge\left(j_{3}^{*}\right) \wedge\left(j_{1}^{*} \vee j_{2}^{*} \vee j_{3}^{*}\right) \wedge\left(j_{1}^{*} \vee j_{2}^{*} \vee j_{3}^{*}\right) \\
& \wedge\left(j_{3}^{*}\right) \wedge\left(j_{3}^{*}\right) \wedge\left(j_{1}^{*} \vee j_{2}^{*} \vee j_{3}^{*}\right) \wedge\left(j_{1}^{*} \vee j_{3}^{*}\right) \wedge\left(j_{1}^{*} \vee j_{2}^{*} \vee j_{3}^{*}\right) \\
& \wedge\left(j_{3}^{*}\right) \wedge\left(j_{1}^{*} \vee j_{2}^{*} \vee j_{3}^{*}\right) \wedge\left(j_{1}^{*} \vee j_{3}^{*}\right) \wedge\left(j_{1}^{*} \vee j_{2}^{*} \vee j_{3}^{*}\right) \wedge\left(j_{1}^{*} \vee j_{2}^{*} \vee j_{3}^{*}\right) \\
& \wedge\left(j_{1}^{*} \vee j_{2}^{*} \vee j_{3}^{*}\right) \wedge\left(j_{1}^{*} \vee j_{2}^{*} \vee j_{3}^{*}\right) \wedge\left(j_{1}^{*} \vee j_{2}^{*} \vee j_{3}^{*}\right) \wedge\left(j_{1}^{*} \vee j_{2}^{*} \vee j_{3}^{*}\right) \wedge\left(j_{1}^{*} \vee j_{2}^{*} \vee j_{3}^{*}\right) .
\end{aligned}
$$

Where $\vee$ and $\wedge$ indicates $\max$ and min,respectively. Thus,

$$
\begin{aligned}
f\left(j_{1}^{*}, j_{2}^{*}, j_{3}^{*}\right) & =\left(j_{1}^{*} \vee j_{2}^{*} \vee j_{3}^{*}\right) \wedge\left(j_{1}^{*} \vee j_{2}^{*}\right) \wedge\left(j_{1}^{*} \vee j_{3}^{*}\right) \\
& =\left(j_{1}^{*} \wedge j_{3}^{*}\right) \vee\left(j_{2}^{*} \wedge j_{3}^{*}\right)
\end{aligned}
$$

Then the reduct of $C$ are $\left\{j_{1}, j_{3}\right\}$ and $\left\{j_{2}, j_{3}\right\}$. As we know that core $(j)=\left\{j_{3}\right\}$ by visual inspection. Therefore,

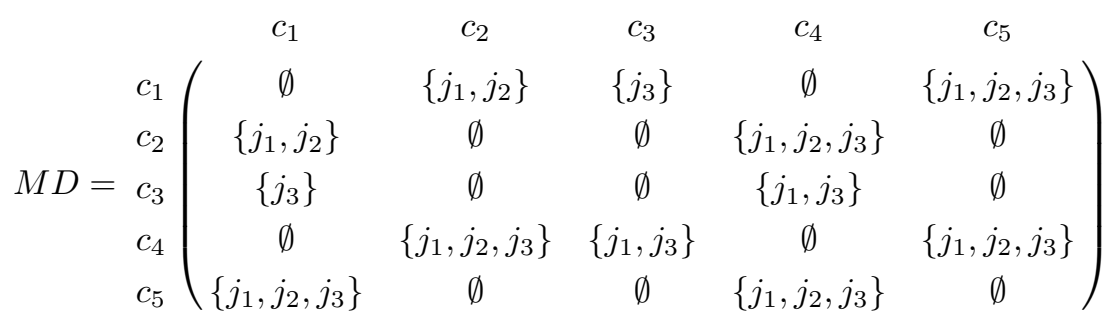

where the discernibility function is as follows:

$$
\begin{aligned}
f_{d}\left(j_{1}^{*}, j_{2}^{*}, j_{3}^{*}\right) & =\left(j_{1}^{*} \vee j_{2}^{*}\right) \wedge\left(j_{3}^{*}\right) \wedge\left(j_{1}^{*} \vee j_{2}^{*} \vee j_{3}^{*}\right) \wedge\left(j_{1}^{*} \vee j_{2}^{*}\right) \wedge\left(j_{1}^{*} \vee j_{2}^{*} \vee j_{3}^{*}\right) \wedge\left(j_{3}^{*}\right) \\
& \wedge\left(j_{1}^{*} \vee j_{3}^{*}\right) \wedge\left(j_{1}^{*} \vee j_{2}^{*} \vee j_{3}^{*}\right) \wedge\left(j_{1}^{*} \vee j_{3}^{*}\right) \wedge\left(j_{1}^{*} \vee j_{2}^{*} \vee j_{3}^{*}\right) \wedge\left(j_{1}^{*} \vee j_{2}^{*} \vee j_{3}^{*}\right) \\
& \wedge\left(j_{1}^{*} \vee j_{2}^{*} \vee j_{3}^{*}\right)
\end{aligned}
$$

Thus,

$$
\begin{aligned}
f_{d}\left(j_{1}^{*}, j_{2}^{*}, j_{3}^{*}\right) & =\left(j_{1}^{*} \vee j_{2}^{*}\right) \wedge\left(j_{3}^{*}\right) \wedge\left(j_{1}^{*} \vee j_{2}^{*} \vee j_{3}^{*}\right) \wedge\left(j_{1}^{*} \vee j_{3}^{*}\right) \\
& =\left(j_{1}^{*} \wedge j_{3}^{*}\right) \vee\left(j_{2}^{*} \wedge j_{3}^{*}\right)
\end{aligned}
$$

Then have the relative reducts of $C,\left\{j_{1}, j_{3}\right\}$ and $\left\{j_{2}, j_{3}\right\}$. We can also identify $\operatorname{core}_{d}(j)=\left\{j_{3}\right\}$ by visual inspection. 


\section{Conclusion}

In this paper we explores the ways for data reduction by using of rough and fuzzy sets theory. we reviewed the most important methods and then investigate the problems of these methods. Also, in current study we present a method to data reduction based on rough and fuzzy sets theory. A numerical example is examined using the proposed method.

\section{References}

[1] An, S., Hu, Q., Pedrycz, W., Zhu, P. and Tsang, E. C. C. (2016). Data-distribution-aware fuzzy rough set model and its application to robust classification. IEEE Transactions on Cybernetics, 46(12), 3073-3085. doi: 10.1109/TCYB.2015.2496425

[2] Banerjee, M. and Pal, S. K. (1996). Roughness of a fuzzy set. Information Science, 93(3-4), 235-246. doi: 10.1016/0020-0255(96)00081-3

[3] Beynon, M. (2001). Reducts with the variable precision rough sets model: A further investigation. European Journal of Operational Research, 134(3), 592-605. doi: 10.1016/S0377-2217(00)00280-0

[4] Chen, D., Kwong, S., He, Q. and Wang, H. (2012). Geometrical interpretation and applications of membership functions with fuzzy rough sets. Fuzzy Sets and Systems, 193, 122-135. doi: 10.1016/j.fss.2011.07.011

[5] Dai, J. and Tian, H. (2013). Fuzzy rough set model for set-valued data. Fuzzy Sets and Systems, 229, 54-68. doi: 10.1016/j.fss.2013.03.005

[6] Dubois, D. and Prade, H. (1990). Rough fuzzy sets and fuzzy rough sets internat. International Journal of General Systems, 17(2-3), 191-209. doi: 10.1080/03081079008935107

[7] Dubois, D. and Prade, H. (1992). Putting fuzzy sets and rough sets together. In Slowinski, R. (Ed.), Intelligent Decision Support (pp. 203-232). Dordrecht: Springer. doi: 10.1007/978-94-015-79759_14

[8] Gorzalczany, B. (1988). Interval-valued fuzzy controller based on verbal model of object. Fuzzy Sets and Systems, 28(1), 45-53. doi: 10.1016/0165-0114(88)90115-7

[9] Guan, Y. Y. and Wang, H. (2006). Set-valued information systems. Information Science, 176(17), 2507-2525. doi: 10.1016/j.ins.2005.12.007

[10] Huang, B., Guo, C. X., Zhuang, Y. L., Li, H. X. and Zhou, X. Z. (2014). Intuitionistic fuzzy multigranulation rough sets. Information Sciences, 277, 299-320. doi: 10.1016/j.ins.2014.02.064

[11] Jensen, R. and Shen, Q. (2004). Fuzzy rough attribute reduction with application to web categorization. Fuzzy Sets and Systems, 141(3), 469-485. doi: 10.1016/S0165-0114(03)00021-6

[12] Jensen, R. and Shen, Q. (2005). Fuzzy-rough data reduction whit and colony optimization. Fuzzy Sets and Systems, 149(1), 5-20. doi: 10.1016/j.fss.2004.07.014

[13] Kondo, M. (2006). On the structure of generalized rough set. Information Science, 176(5), 589600. doi: 10.1016/j.ins.2005.01.001

[14] Kryszkiewicz, M. (1998). Rough set approach to incomplete information systems. Information Science, 112(1-4), 39-49. doi: 10.1016/S0020-0255(98)10019-1

[15] Kryszkiewicz, M. (1999). Rules in incomplete information system. Information Science, 113(3-4), 271-292. doi: 10.1016/S0020-0255(98)10065-8

[16] Liang, J., Wang, F., Dang, C. and Qian, Y. (2014). A group incremental approach to feature selection applying rough set technique. IEEE Transactions on Knowledge and Data Engineering, 26(2), 294-308. doi: 10.1109/TKDE.2012.146

[17] Lin, Y., Hu, Q., Liu, J., Li, J. and Wu, X. (2017). Streaming feature selection for multilabel learning based on fuzzy mutual information. IEEE Transactions on Fuzzy Systems, 25(6), 14911507. doi: 10.1109/TFUZZ.2017.2735947

[18] Lin, T. Y. and Liu, Q. (1994). Rough approximate operators: Axiomatic rough set theory. In Ziarko, W. P. (Ed), Proceedings of the International Workshop on Rough Sets and Knowledge - Rough Sets, Fuzzy Sets and Knowledge Discovery (pp. 256-260). Berlin: Springer. doi: 10.1007/978-14471-3238-7_31

[19] Lin, Y., Li, Y., Wang, C. and Chen, J. (2018). Attribute reduction for multi-label learning with fuzzy rough set. Knowledge-Based Systems, 152, 51-61. doi: 10.1016/j.knosys.2018.04.004 
[20] Nachtegael, M. and Kerre, E. E. (1998). The dizzy number of fuzzy implication operators on finite chains. In Ruan, D., Abderrahim, H. A., D'hondt, P. and Kerre, E. E. (Eds.), Proceedings of the 3rd International FLINS Workshop - Fuzzy Logic and Intelligent Technologies for Nuclear Science and Industry. Singapure. doi: 10.1142/3845

[21] Pawlak, Z. (1982). Rough sets. International Journal of Computer and Information Sciences, 11(5), 341-356. doi: 10.1007/BF01001956

[22] Pawlak, Z. and Skowron, A. (2007). Raudiments of rough set. Information Sciences, 177(1), 3-27. doi: $10.1016 /$ j.ins.2006.06.003

[23] Pawlak, Z. and Skowron, A. (2007). Rough sets: Some extensions. Information Sciences, 177(1), 28-40. doi: 10.1016/j.ins.2006.06.006

[24] Pawlak, Z. (1991). Rough sets: Theoretical aspects of reasoning about data. Dordrecht: Springer. doi: 10.1007/978-94-011-3534-4

[25] Qian, Y. H., Dang, C., Liang, J. Y. and Tang, D. (2009). Set-valued order information system. Information Science, 179(16), 2809-2832. doi: 10.1016/j.ins.2009.04.007

[26] Radzikowska, A. M. and Kerre, E. E. (2002). A comparative study of fuzzy rough sets. Fuzzy Sets and Systems, 126(2), 137-155. doi: 10.1016/s0165-0114(01)00032-x

[27] Richard, J. and Qiang, SH. (2002). Fuzzy-rough sets for descriptive dimensionality reduction. Proceedings of the IEEE International Conference on Fuzzy Systems, 1, 29-34. doi: 10.1109/fuzz.2002.1004954

[28] Sun, B., Gong, Z. and Chen, D. (2008). Fuzzy rough set for the interval-valued fuzzy information systems. Information Science, 178(13), 2794-2815. doi: 10.1016/j.ins.2008.03.001

[29] Sun, B., Ma, W. and Chen, X. (2015). Fuzzy rough set on probabilistic approximation space over two universes and its application to emergency decision-making. Expert Systems, 32(4), 507-521. doi: 10.1111/exsy.12103

[30] Sun, B., Ma, W. and Qian, Y. (2017). Multigranulation fuzzy rough set over two universes and its application to decision making. Knowledge-Based Systems, 123, 61-74. doi: 10.1016/j.knosys.2017.01.036

[31] Wang, C., Huang, Y., Shao, M. and Fan, X. (2019). Fuzzy rough set-based attribute reduction using distance measures. Knowledge-Based Systems, 164, 205-212. doi: 10.1016/j.knosys.2018.10.038

[32] Wang, C., Qi, Y., Shao, M., Hu, Q., Chen, D., Qian, Y. and Lin, Y. (2017). A Fitting Model for Feature Selection with Fuzzy Rough Sets. IEEE Transactions on Fuzzy Systems, 25(4), 741-753. doi: 10.1109/TFUZZ.2016.2574918

[33] Xu, J., Wang, Y., Xu, K. and Zhang, T. (2019). Feature genes selection using fuzzy rough uncertainty metric for tumor diagnosis. Computational and Mathematical Methods in Medicine, 1-9. doi: 10.1155/2019/6705648

[34] Yang, Y., Chen, D., Wang, H., Tsang, E. C. C. and Zhang, D. (2017). Fuzzy rough set based incremental attribute reduction from dynamic data with sample arriving. Fuzzy Sets and Systems, 312, 66-86. doi: 10.1016/j.fss.2016.08.001

[35] Zhang, H. G., Liang, H. L. and Liu, D. R. (2004). Two new operators in rough set theory with application to fuzzy sets. Information Science, 166(1-4), 147-165. doi: 10.1016/j.ins.2003.11.003

[36] Zhao, S., Chen, H., Li, C., Du, X. and Sun, H. (2015). A novel approach to building a robust fuzzy rough classifier. IEEE Transactions on Fuzzy Systems, 23(4), 769-786. doi: 10.1109/TFUZZ.2014.2327993 